\title{
A novel deep learning scheme for morphology- based classification of mycobacterial infection in unstained macrophages
}

\author{
Xinzhuo Zhao, Yanqing Bao, Lin Wang, Wei Qian and Jianjun Sun*
}

\begin{abstract}
Objective: Mycobacterium tuberculosis (Mtb) is an airborne, contagious bacterial pathogen that causes widespread infections in humans. Using Mycobacterium marinum (Mm), a surrogate model organism for Mtb research, the present study develops a deep learning-based scheme that can classify the Mminfected and uninfected macrophages in tissue culture solely based on morphological changes. Methods: A novel weak- and semisupervised learning method is developed to detect and extract the cells, firstly. Then, transfer learning and fine-tuning from the CNN is built to classify the infected and uninfected cells. Results: The performance is evaluated by accuracy (ACC), sensitivity (SENS) and specificity (SPEC) with 10-fold cross-validation. It demonstrates that the scheme can classify the infected cells accurately and efficiently at the early infection stage. At 2 hour post infection (hpi), we achieve the ACC of $0.923 \pm 0.005$, SENS of $0.938 \pm 0.020$, and SPEC of $0.905 \pm 0.019$, indicating that the scheme has detected significant morphological differences between the infected and uninfected macrophages, although these differences are hardly visible to naked eyes. Interestingly, the ACC at 12 and $24 \mathrm{hpi}$ are $0.749 \pm 0.010$ and $0.824 \pm 0.009$, respectively, suggesting that the infection-induced morphological changes are dynamic throughout the infection. Finally, deconvolution with guided propagation maps the key morphological features contributing to the classification. Significance: This proof-ofconcept study provides a novel venue to investigate bacterial pathogenesis in a macroscopic level and has a great promise in diagnosis of bacterial infections.
\end{abstract}

Index Terms - cell classification, cell detection, deep learning, infection detection, mycobacterial infection, tuberculosis

\section{INTRODUCTION}

$\mathrm{M}$ YCOBACTERIUM is a genus of Actinobacteria [1], which includes pathogens known to cause serious diseases in humans, such as Mycobacterium tuberculosis (Mtb) that causes global tuberculosis epidemic and accounts for more than 15 millions of deaths since 2000 [2]. Early and accurate detection of Mtb infection is an urgent need. Traditional diagnosis of TB relies on recovery of live Mtb organisms from patients' sputum samples by microbiological culture, but this method is time consuming and has a low detection rate [3].

Research reported in this publication was supported by the National Institute Of General Medical Sciences of the National Institutes of Health under Award Number SC1GM095475 (to J. Sun), National Center for Research Resources (5G12RR008124); and National Institute on Minority Health and Health Disparities (G12MD007592).. The content is solely the responsibility of the authors and does not necessarily represent the official views of the National Institutes of Health; Xinzhuo Zhao was with Northeastern University, Shenyang, China. She is now with the Department of Biological Sciences and
Recently, various molecular and immunological methods have been developed for TB diagnosis, including sputum smear microscopy [4][5], tuberculin skin test [6], interferon- $\gamma$ release assays [7], and Xpert MTB/RIF [8], etc. Although each of these methods has unique advantages and has been used in some clinical settings, none of them can directly detect live Mtb organisms from patients. Thus the traditional microbiological culture of live Mtb from patients remains as the well-accepted gold standard for TB diagnosis.

Image analysis has a great potential in disease diagnosis. Large amounts of advanced quantitative features from images are extracted and analyzed, and the image data are processed in a mineable form to build descriptive and predictive models correlating image features to specific diseases even with geneprotein signatures [9]. For instances, medical image analysis has been able to distinguish benignant lung nodules from the malignant ones by the CT scan images [10][11]. Breast tumors can be detected and analyzed automatically based on the radiological images [12]-[14]. The brain partitions have been segmented for further exploration [15][16]. Image analysis is also extensively involved in basic biomedical sciences and biology. For instances, the cell organs are segmented [17] and cellular classification tasks are also manipulated by image analysis [18].

There are two main challenges for the image analysis: feature extraction and the choice of the classifier. For the feature extraction, deep learning, especially the convolutional neural network (CNN), has made a dramatic progress [19]. Compared with traditional hand-crafted features, $\mathrm{CNN}$ extracts ample features automatically. In natural images classification task, CNN has achieved superhuman performance [20]-[22]. Generally, the CNN model can be generated by training from scratch or from the transfer learning method. Transfer learning means to utilize the knowledge learned from one task to another task, as long as the two tasks share the same features [23]. Compared with training the CNN model from scratch, transfer learning involves less manual modifications and less input images. It also helps to enhance the classification performance and reduce the training time. For the classifier, there are three

Border Biomedical Research Center, University of Texas at El Paso, TX, USA. Yanqing Bao, Lin Wang, Jianjun Sun are with the Department of Biological Sciences and Border Biomedical Research Center, University of Texas at El Paso, TX, USA. Wei Qian is with Department of Electrical and Computer Engineering, University of Texas at El Paso, TX, USA. (correspondence e-mail: jsun@utep.edu). 
kinds of machine learning methods: unsupervised, semisupervised, and supervised learning. Unsupervised learning trains the predictive model with unlabeled data. For example, images features can be related to gene-mutation by unsupervised learning without any prior knowledge [24]. Supervised learning generally obtains more accurate results, owning to its abundant labeled data training process. Semisupervised learning falls between, which requests only a small amount of labeled data with a large amount of unlabeled data. Thus, it combines the advantages of unsupervised and supervised learning methods.

Considering the significant success of image analysis in medical and biological sciences, we are inspired to introduce $\mathrm{CNN}$ to the classification task of the microscopic world. In this study, we systematically design a scheme to distinguish mycobacteria-infected cells from uninfected cells based on cellular morphological changes that are indistinguishable to naked eyes at the early stage of infection. First, a weak- and semi-supervised method is developed to detect cells from the images. It combines the gradient-based circle Hough transform (GBCHT) algorithm [25] with the supervised deep learning. Second, the cells are classified by transfer learning and finetuning. Third, image reconstruction by deconvolution is applied to detect the key morphological features contributing to the classification results.

\section{Materials AND MethodS}

\section{A. Data Acquisition}

Mycobacterium marinum $(\mathrm{Mm})$ is a pathogen that causes tuberculosis-like diseases in fish and sometimes causes mild skin infections in human. Since Mm shares similarities with $\mathrm{Mtb}$, in genomes, virulence factors and modes of infection in macrophages, it is widely accepted as a safer surrogate model for Mtb in laboratory research. Here, we use a Mm strain harboring a pMSP12:mCherry plasmid (Addgene, USA), which expresses a mCherry protein so that the bacteria emit cherry fluorescence [26]. Thus, the cherry fluorescence serves as an infection marker to train $\mathrm{CNN}$ and also to validate the classification results.

At the early stage of Mtb infection, alveolar macrophage is the first phagocyte that recognizes and engulfs Mtb. The murine macrophage cell line RAW264.7 is one of the most commonly used cell line for the studies of intracellular infection by Mtb and Mm. Thus, this study uses RAW264.7 cells as the infected host model.

RAW264.7 cells are plated in 8-well chambered culture slides (Corning Falcon, USA) at $1 \times 10^{5}$ cells/well and cultured in Dulbecco's modified eagle medium (DMEM, Hyclone, USA) containing $10 \%$ fetal bovine serum (FBS, Corning, USA) at $37^{\circ} \mathrm{C}, 5 \% \mathrm{CO} 2$ for $24 \mathrm{hr}$. Cells are washed three times with prewarmed PBS to remove residual antibiotics. Then, the Mm bacteria expressing mCherry are added into the wells to reach 2 multiplicity of infection (MOI), followed by centrifugation at $250 \times \mathrm{g}$ at room temperature (RT) for $5 \mathrm{~min}$. After $30 \mathrm{~min}$ incubation at $30^{\circ} \mathrm{C}$, the cells are washed again with pre-warmed PBS to remove unbound bacteria. Then DMEM with $100 \mu \mathrm{g} / \mathrm{ml}$
Amikacin is added to kill the extracellular bacteria for $1 \mathrm{hr}$ at $30^{\circ} \mathrm{C}$. The infected cells are cultured in DMEM containing $1 \%$ FBS, $50 \mu \mathrm{g} / \mathrm{ml}$ Amikacin at $30^{\circ} \mathrm{C}, 5 \% \mathrm{CO}_{2}$ until image acquisition.

At 2, 12 and 24 hours post infection (hpi), the cells are washed with PBS and fixed with $4 \%$ paraformaldehyde for $10 \mathrm{~min}$ at RT. Once the fixed slides are dried in air, mount media (Enzo Life Sciences, USA) is added to prevent fluorescence quench. Finally, the slides are covered with cover slips. The slides are imaged with a FLoid Cell Imaging Station (Life technologies, USA) at $460 \mathrm{X}$ magnification with the white light channel to image the cells and the red fluorescence channel to image the cherry color bacteria. The images were exported as 16-bit tagged image file format (TIFF) with a final resolution of 96 digital pixels per inch (dpi).

\section{B. Cell Detection}

All of the image processing and analysis are performed using MATLAB language and Caffe [27] framework in a workstation with a GTX1060 GPU.

A novel weak- and semi-supervised detection (WSSD) algorithm is developed for cell detection. This method combines the gradient-based circular Hough transform (GBCHT) [25] with the convolutional neural network. GBCHT is a feature-based learning method and doesn't need manually labeled input data. $\mathrm{CNN}$ is a supervised learning algorithm. Our novel algorithm uses a small amount of manually labeled data along with a large amount of unlabeled data, which is therefore named as weak- and semi-supervised detection (WSSD) algorithm.

Hough transform [28] was put forward to detect specific line patterns. When detecting circles, the points in the images are transformed to the parameter space, seen as (1):

$$
r^{2}=(x-a)^{2}+(y-b)^{2}(1)
$$

leading the point represented by three parameters: $r, a$ and $b$. Where $r$ is the radius of each detected circle, and $a$ and $b$ present the location of the center of the circle. To improve the detection accuracy, gradient is utilized in [25][29]. First, the gradient to the center of the whole image is calculated by (2):

$$
\nabla I_{i, j}=\left.\left(V_{x}, V_{y}\right)\right|_{i, j}=\left(I_{i, j}-I_{i, j-1}, I_{i, j}-I_{i-1, j}\right)
$$

where $(i, j)$ indices the pixel position. $I_{i, j}$ is the intensity of the pixel. $\nabla I_{i, j}$ is the gradient vector of the pixel at the position $(i$, $j)$. Then, an accumulation step is introduced to find the highest response of the gradient, which would be the center of the circle. For the determination of the radii [30], if the point satisfies (3), where $\Delta r$ is the interval between adjacent $r$ values.

$$
|||q(i, j) \|-r|<\Delta r / 2 \text { (3) }
$$

Besides cell detection, small amounts of cells are labeled as the positive or negative cells in instance-level (i.e., bounding boxes), as shown in Fig. 1. Instead of classifying the cells in the image-level labels, it's preferred to distinguish which single cell is infected. Because in the images of the infected group, not all of the cells are infected. Then, these labeled cells are used to train a classifier to discriminate the true infected cells from the uninfected cells. Transfer learning and fine-tuning from the CNN are utilized, which will be introduced in next subsection. 


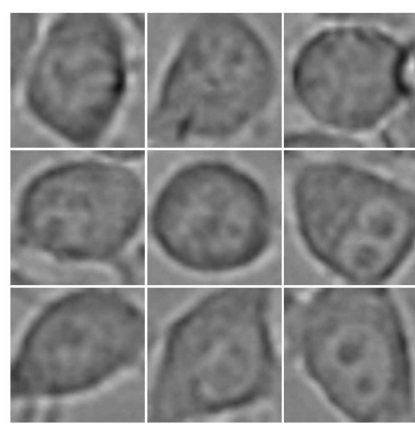

(a)

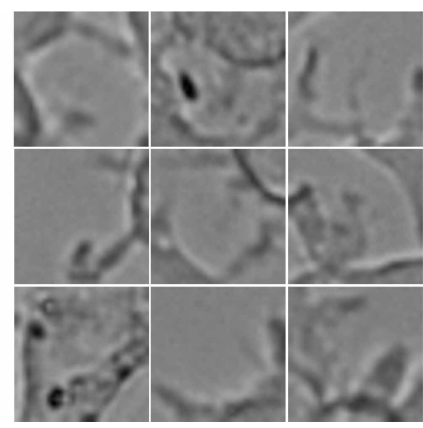

(b)
Fig. 1. Manually labeled cells of the GBCHT detection. The cells are initially detected from the whole images by the GBCHT algorithm. Considering there being miss detected or error detected cells, the detected cells are labeled as the positive cells, shown in (a), and the negative cells, shown in (b). The labeled cells are then utilized for further supervised learning process.

After the classifier is well trained, the rest of the cells, which are the unlabeled ones, are classified by the prediction model.

\section{Transfer Learning and Fine-tuning for Classification}

This is a binary classification task. There are two experimental groups: the control group, in which all the cells are not infected; the infection group, which contains infected cells and uninfected cells since the rate of infection is less than $100 \%$. The cells in control group are extracted directly by WSSD as the labeled uninfected cells. For the infection group, only the cells with cherry color bacteria either within or on the circles are considered as infected cells and extracted from the images, as seen in Fig. 2.

1) Convolutional neural network architecture

ResNet [31], a contemporary CNN architecture, is one of the best deep learning classifier with 152 layers. It is designed for $224 \times 224$ pixel images. In view of its deep structure, the memory of GPU can't meet the computational cost. Thus, a cropped version with ResNet-50 [32] is utilized in this experiment. Seen in Table I, it's a stack of 5 groups of convolutional layers. Each group consists of several residual blocks. It adds on a "delta" or a slight change to the original input, called "residual".

2) Transfer learning and fine-tuning from $C N N$

There are three methods to implement transfer learning and fine-tuning. (1) Only transfer learning without fine-tuning. The trained deep learning model is directly used for prediction without adjustment by the new task images. This method is underperforming. (2) Transfer learning with fine-tuning of the

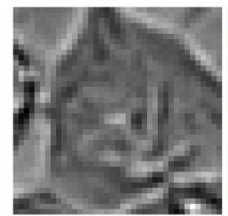

(a)

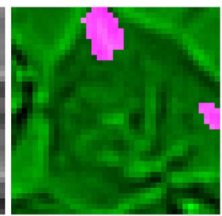

(b)

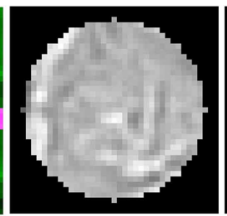

(c)

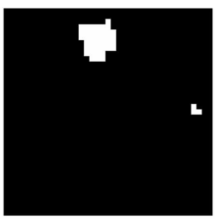

(d)
Fig. 2. Extraction of the Mm-infected cells. Since the rate of infection is less than $100 \%$, extra filter is applied to remove the cells without mCherry fluorescence (regarded as uninfected cells). (a) an infected cell in the view of white light; (b) the merge of mCherry fluorescence from bacteria with the white light images; (c) the mask for the cell; (d) the overlapping region between the cell and the bacteria.
TABLE I

THE STRUCTURE OF THE TRANSFER LEARNING AND FINE-TUNING FROM THE RESNET-50

\begin{tabular}{cccc}
\hline \hline Layer & Layer setting & $\begin{array}{c}\text { Kernel } \\
\text { Number }\end{array}$ & Output Size \\
Name & & & $224 \times 224$ \\
\hline Input & & 64 & $64 \times 112 \times 112$ \\
Conv1 & $7 \times 7, p 3, s 2$ & & $64 \times 56 \times 56$ \\
Pooling & max,3 3 3,s2 & & \\
Conv2_x & {$\left[\begin{array}{c}1 \times 1, * p 0, * s 1 \\
3 \times 3, p 1, s 1 \\
1 \times 1, p 0, s 1\end{array}\right] \times 3$} & {$\left[\begin{array}{c}64 \\
64 \\
256\end{array}\right] \times 3$} & $256 \times 56 \times 56$ \\
& {$\left[\begin{array}{c}1 \times 1, * p 0, * s 1 \\
3 \times 3, p 1, s 1 \\
1 \times 1, p 0, s 1\end{array}\right] \times 4$} & {$\left[\begin{array}{c}128 \\
128 \\
512\end{array}\right] \times 4$} & $512 \times 28 \times 28$ \\
Conv3_x & {$\left[\begin{array}{c}1 \times 1, * p 0 *, s 1 \\
3 \times 3, p 1, s 1 \\
1 \times 1, p 0, s 1\end{array}\right] \times 6$} & {$\left[\begin{array}{c}256 \\
256 \\
1024\end{array}\right] \times 6$} & $1024 \times 14 \times 14$ \\
Conv4_x & {$\left[\begin{array}{c}1 \times 1, * p 0, * s 1 \\
3 \times 3, p 1, s 1 \\
1 \times 1, p 0, s 1\end{array}\right] \times 3$} & {$\left[\begin{array}{c}512 \\
512 \\
2048\end{array}\right] \times 3$} & $2048 \times 7 \times 7$ \\
Conv5_x & Ave,2 classes & & \\
Pooling & Loss & & $2048 \times 1 \times 1$ \\
\hline \hline
\end{tabular}

$p$ is short for pad; $s$ is short for stride.

* means its labeled parameters are not constant. For each convolutional layer, it consists several residual blocks. In Conv2_x, Conv3_x, and Conv4_x layer, the input images are down sampled by the first time of convolutional computation. Thus, the pad is 1 and stride is 2 in the first convolutional computation of the first residual block in these three layers.

last classification layer. For example, the trained model is based on the ImageNet that classifies the 1000 classes. The new task just needs to distinguish between 10 classes. This method needs to initialize the parameters of the last classification layer and to change the output class into 10 . The former convolutional layers do not participate the backpropagation, which will not be fine-tuned. Compared with the other two methods, this one performs in between. (3) Transfer learning with fine-tuning of all the layers, including the convolutional layers. It means all the weights inherited from the trained deep learning model are fine-tuned by the new task datasets and all layers participate the backward computation. This one outperforms the other two [33]. In our experiment, the third method, which performs the best, is utilized.

At the beginning, the pre-trained network is trained by the natural images with random initialized weights. Then, the pretrained model is transferred to Mm infection classification task. The weights and bias of the last fully connected layers are initialized randomly. The neural units are reduced to two. None of any layers in the entire network have frozen the weights and bias. All of them are fine-tuned by backward computation. The learning rates are changed to 0.0005 , which is far smaller than their original one.

\section{Deconvolution}

Deconvolution is a method for visualizing the features learned by the hidden units of CNNs. It's also named as transposed convolution or backward stride convolution[34]. In 
deep learning, it is an inverse flow of its original training process. Given a high level feature map, by the backward computation, the reconstructed image will show the strongly activated part of the input images. By deconvolution, the hidden layers of deep neural network can be observed by eyes. Zeiler introduced a novel visualization technique that gave insights into the function of intermediate feature layers and analyzed the strongest activation of the input images by deconvolutional network. The observation of the deconvolutional results is improved by the guided backpropagation [35].

In this experiment, guide backpropagation is performed to reconstruct image. In the training process, the input images are down sampled by several convolutional layers and pooling layers. Therefore, when reconstructing the images, two up sampling ways are proceeded. By adjusting the stride steps or padding numbers, the reconstructed images can be up sampled. The details are well described in [36]. Beside backward stride convolution, up-pooling is another key step to affect the final reconstruction result. There are three main methods for reconstruction: back propagation, deconvnet and guide backpropagation. When doing the ReLU of forward propagation, the position of eliminated pixels (less than zero) will be recorded. In back propagation, the same position of the pixels will still be eliminated from the backpropagation, regardless of its value being less than zero or not. In deconvnet [37], the position of eliminated pixels will be reevaluated by the actual value (whether is less than zero). Guided backpropagation combines these two methods, which removes both of the values eliminated by the last two ones. The deconvolution reconstruction is based on the DeepVis Toolbox [37].

\section{RESULTS}

\section{A. The Comparison between WSSD and GBCHT}

The cell detection results of GBCHT are shown in Fig. 3. The original cell image is exhibited in Fig. 3a and the accumulation array of each pixel's gradient is shown in Fig. 3b. By searching the local maxima, the center of the circle can be detected. Although, not all the cells are regular circular, both circular and irregular shapes can still be observed in the experiment, as seen in Fig. 3c. The red cross in the middle of the image is supposed to be the circle center. Besides the irregular shapes, the

TABLE II

THE SUMMARY OF THE DETECT RESULTS

\begin{tabular}{ccccc}
\hline \hline \multirow{2}{*}{ Term } & Equation & $\begin{array}{c}\text { Result of } \\
\text { GBCHT }\end{array}$ & $\begin{array}{c}\text { Result of } \\
\text { WSSD }\end{array}$ & $p$-value \\
\hline \multirow{2}{*}{ Accuracy } & $\frac{T P+T N}{T P+F P+T N+F N}$ & $0.843 \pm 0.021$ & $\mathbf{0 . 9 1 2} \pm \mathbf{0 . 0 3 3}$ & 0.034 \\
\cline { 2 - 5 } Precision & $\frac{T P}{T P+F P}$ & $0.898 \pm 0.039$ & $\mathbf{0 . 9 9 4} \pm \mathbf{0 . 0 0 9}$ & 0.016 \\
& $\frac{T P}{T P+F N}$ & $\mathbf{0 . 9 3 4} \pm \mathbf{0 . 0 2 4}$ & $0.907 \pm 0.034$ & 0.297 \\
Sensitivity & $\frac{}{T P+}$ & & & \\
\hline \hline
\end{tabular}

There is significant difference between two models if $\mathrm{p}<0.05$.

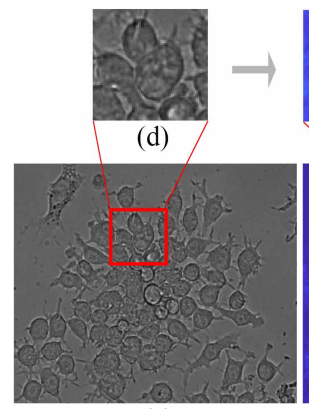

(a)

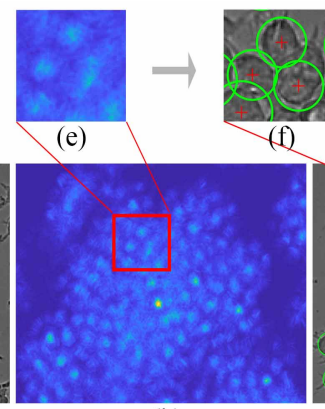

(b)

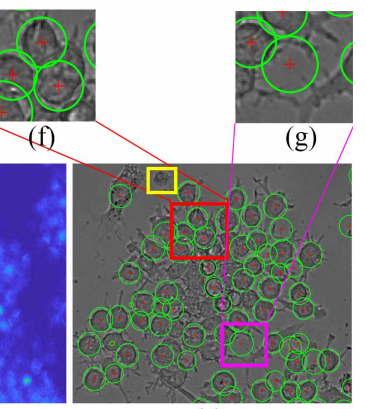

(c)
Fig. 3. Cell detection results with gradient-based circular Hough transform. (a) The original image taken by white light channel; (b) The accumulation array of each pixel's gradient. The high response points are the detected centers of the circles; (c) The detection results with each green circle indicating a cell. The yellow square shows a cell that is missed in detection. To observe the details, parts of the images are enlarged and shown as subgraphs. (d) - (e) The example of the overlapped cells. (g) The error detected example.

algorithm is also robust to the overlapped cells, as seen in Fig. $3 \mathrm{~d}$. They are also correctly detected in Fig. 3f. To evaluate the detection performance, quantitative assessment is implement. Some cells are correctly detected (Fig. 3f) and named as true positive (TP). The yellow squared cell shown in the Fig. $3 \mathrm{c}$ is a cell that is missed in detection, named as false negative (FN). Besides those, some non-cell regions were detected as cells by error, named as false positive (FP), as seen in Fig. 3g.

To further improve the detection performance, WSSD is developed. We use the same images to evaluate the performance of WSSD, for the comparison between GBCHT. The statistical results are shown in Table II. It's obvious that there are significant improvements of the new method in accuracy and precision with $p<0.05$. According to the results, this new method reduces the false positive. Granted that, the WSSD's sensitivity is not as high as GBCHT's, meaning more real cells are misidentified as the background, but it can still ensure that the $99.4 \%$ remaining cells are the true positive ones.

\section{B. Evaluation of the Cell Classification}

Transfer learning and fine-tuning from ResNet are used to distinguish the uninfected cell from the Mm-infected cells at 2, 12, and 24 hpi. Fig. 4 presents the training loss and the validation accuracy at 2 hpi. The training loss is reduced with the iteration. And the validation accuracy increases quickly

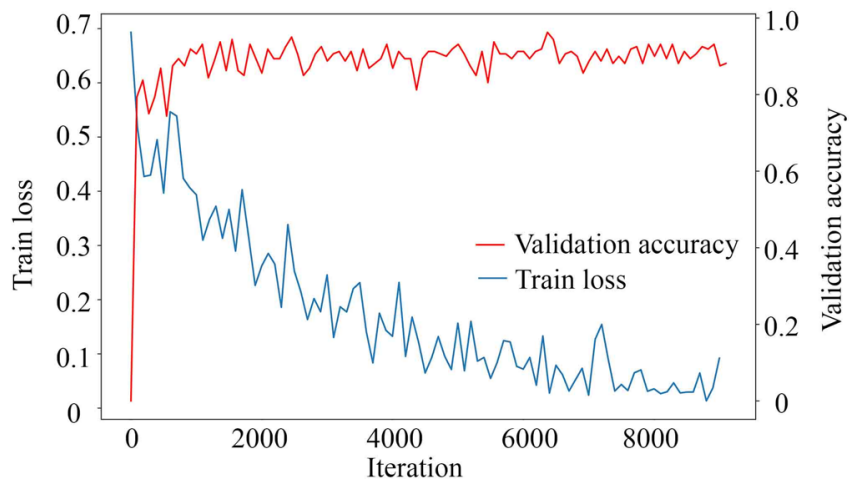

Fig. 4. The train loss and validation accuracy of the transfer learning. The blue line presents the training loss changing with the iteration. The red line shows the validation accuracy. 


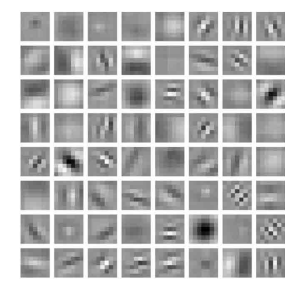

(a)

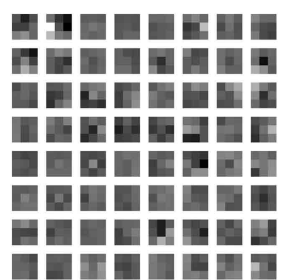

(b)

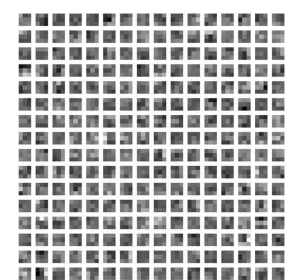

(c)
Fig. 5. Feature examples of the three layers from the trained CNN for classification. (a) is the features from the conv1 layer; (b) and (c) are features of conv2 (layer_64_2_conv2) and conv4 (layer_256_2_conv2) layer. Structured and heterogeneous patterns indicate a well-trained $\bar{C} \mathrm{NN}$ model.

with the iteration, which reaches to $90 \%$ average accuracy. To estimate the "health" of the network, feature maps are observed. Three of them are shown in Fig. 5, which are conv1, conv2 (layer_64_2_conv2), and conv4 (layer_256_2_conv2), respectively. These features show the complex invariances learned in the convolutional layers. The lower layer, seen as the Fig. 5a, shows an organized structure pattern, which are understandable to human. These basic textures indicate the network to be qualified to detect the structure edges of the input images. The higher layer, seen as Fig. $5 \mathrm{~b}$ and $5 \mathrm{c}$, shows some incomprehensible features. Their uncorrelated patterns ensure the variation of different channels, which also guarantees a "healthy network"

The performance of classification is evaluated using the 10fold cross-validation. The results are summarized in Table III. It summarizes the total number of each group of cells and the classification results. Seen as the second and the third column, the numbers of two classes of each time are almost balanced. This balance leads no additional bias to the results. The classification results are also exhibited in an intuitional way in Fig. 6. There are distinct differences between the uninfected cells and Mm-infected cells at $2 \mathrm{hpi}$, which achieves the ACC of $0.938 \pm 0.020$. However, at $12 \mathrm{hpi}$, the cells of two classes are difficult to distinguish, achieving the ACC of $0.749 \pm 0.010$. It indicates less difference in morphologies of two classes of cells. At $24 \mathrm{hpi}$, the ACC turns out to be $0.824 \pm 0.009$. SENS and SPEC have the same trends as the ACC.

\section{Guided backpropagation for deconvolution}

Guided backpropagation is utilized to analyze the influence of each pixel to the classification results. The deconvolutional results of the uninfected control cells (Fig. 7a) and infected cells (Fig. 7b) at 2 hpi are presented. Three guided backpropagation

TABLE III

CELL NUMBERS AND THE CLASSIFICATION RESULTS

\begin{tabular}{cccccc}
\hline \hline Time & $\begin{array}{c}\text { Uninfected } \\
\text { cell }\end{array}$ & $\begin{array}{c}\text { Infected } \\
\text { cell }\end{array}$ & ACC & SENS & SPEC \\
& number & number & & & \\
\hline 2 & 3913 & 3416 & $0.923 \pm 0.005$ & $0.938 \pm 0.020$ & $0.905 \pm 0.019$ \\
12 & 3658 & 3159 & $0.749 \pm 0.010$ & $0.831 \pm 0.008$ & $0.652 \pm 0.021$ \\
24 & 7462 & 6961 & $0.824 \pm 0.009$ & $0.839 \pm 0.033$ & $0.823 \pm 0.059$ \\
\hline \hline
\end{tabular}

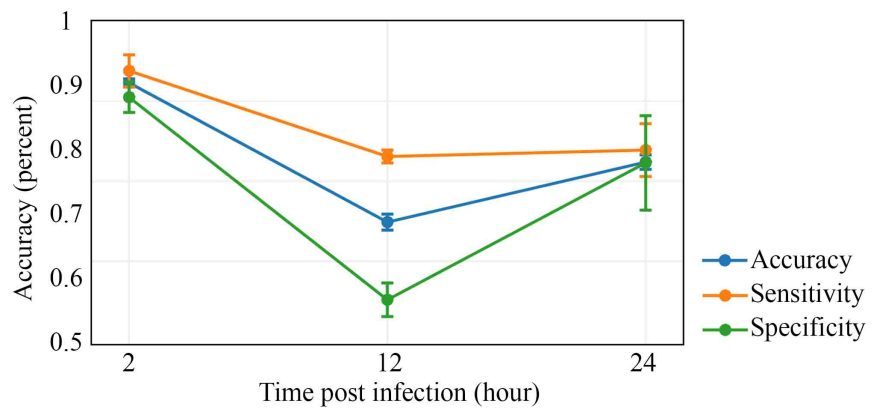

Fig. 6. Classification results of uninfected cells and Mm-infected cells. The cells from infected group or uninfected control group were classified, and Accuracy (blue), Sensitivity (orange) and Specificity (green) were calculated and plotted against the hours of post-infection.

examples from each group are selected because their channels are highly activated, which means they contribute significantly to the decision of the classification. The high active signals in both groups focus on the cell edges, suggesting that cell edges are significant features in the classification of two groups of cells. It is also observed that there are more dots inside the infected cells, such as the bottom right images (Fig. 7b). More interestingly, the high activated pixels have little overlap with the cherry color bacteria, indicating that the CNN does not classify the cells by detecting the bacteria.

\section{Discussion}

This study is the first time that deep learning-based image analysis is used to detect $\mathrm{Mm}$ infection in cultured macrophages. This method detects significant morphological differences between the infected and the uninfected cells as early as $2 \mathrm{hpi}$, even though these changes are indistinguishable to naked eyes. Interestingly, the detection accuracy varies at different times of

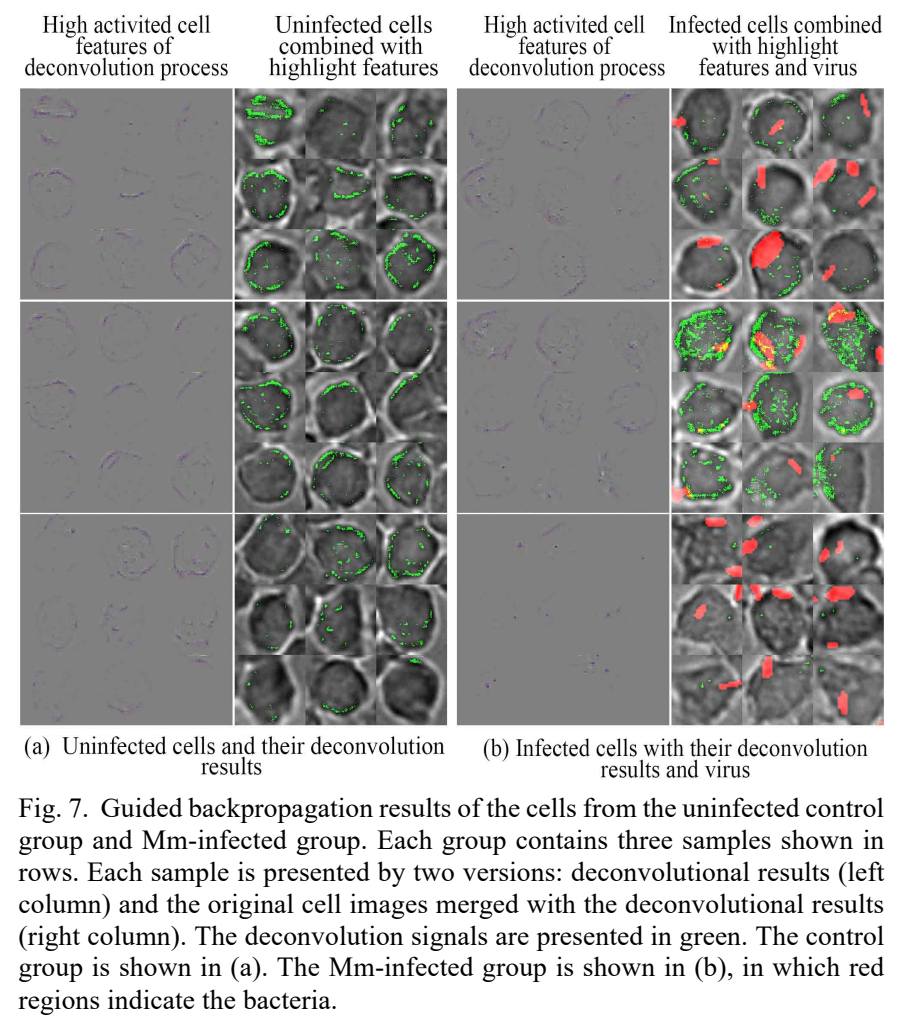


infection. It is high at 2 and $24 \mathrm{hpi}$ and become significantly lower at $12 \mathrm{hpi}$. This result indicates that the host-pathogen interaction is a dynamic process and the infection-induced morphological changes vary accordingly in different infection stages. In fact, it is correlated surprisingly well with the widelyaccepted experimental data that at the early stage of infection, macrophages make strong immune responses to mycobacteria, including engulfing mycobacteria into the phagosomes and activating oxidative stress within the phago-lysosomes [38][39], while at the same time, mycobacteria exert various mechanisms to counteract and suppress the host immune responses, which should be accompanied with significant morphological changes until a balance between host and mycobacteria or a latency is established at the middle stage of the infection [40]-[42]. At the later stage of infection, mycobacteria may break the latency state and transition to active infection, which is evidenced by the observation that $\mathrm{Mm}$ escapes the phagosome into the cytosol, where $\mathrm{Mm}$ replicates, recruits actin tails for cell-to-cell spreading, and eventually causes cell death [43]-[45]. The $\mathrm{CNN}$-detected morphological changes, which are invisible to naked eyes, provide a novel venue to investigate into the mechanism of bacterial pathogenesis.

The newly developed method has three advantages in cell detection: robustness, effectiveness and preciseness. The original GBCHT is widely used in various areas, such as to identify the eyes in facial images [46] or to detect multiple object instances [47]. The developed WSSD doesn't need parameter setting, making it accessible to various tasks. Supervised learning method generally outperforms unsupervised method with the cost of large amount of manual labeled data. For instance, U-Net is a state-of-the-art architecture for detection and segmentation of cells in light microscopy images [48]. Though it achieves good results, it is less favored because of labor consuming. However, in our method, only small amounts of labeled data are needed. The semi-supervised method is efficient because it demands less labeled data. It is also more accurate than the unsupervised method. Currently, this method is only sensitive to quasi-circle objects. Thus, some cells are missed due to irregular shapes, such as extensional pseudopodia. However, it doesn't affect the results as long as enough true positive cells are extracted for analysis, which meet our goal. Taking all these factors into account, the WSSD is well developed in cell detection.

While some cell features are well extracted and analyzed by hand-crafted methods [49]-[51], deep learning has proven to have supreme performance in medical image diagnosis [52]. Xu et al. extracted features of the sickle cell and classified with CNN [53]. Chen et al. also classified the cells by heuristic genetic algorithm using the extracted features [54]. Different from these two works, our raw data are fed directly to the CNN is more sufficient than those designed by human hands. What's more, among the contemporary CNN structures, ResNet outperforms most of the other ones, such as AlexNet [20], VGGNet [21], and GoogLeNet [55]. Therefore, we build our architecture based on the ResNet-50. Proven by our experiment results, the classification accuracy at $2 \mathrm{hpi}$ is as high as $0.923 \pm 0.005$.
The deep learning method accurately detects the Mminduced morphological changes that are indistinguishable to human eyes. It is important to understand where those changes are located and what cellular structures are affected, which will provide clues for future studies to reveal the in-depth mechanism of host-pathogen interaction.

\section{CONCLUSION}

In summary, this study has successfully developed a deep learning-based scheme to classify mycobacteria-infected cells from the uninfected cells. The cells are detected and extracted with a weak- and semi-supervised detection algorithm. Excellent classification accuracy is achieved by the transfer learning and fine-tuning of the CNN. Deconvolution reconstructs the CNN-detected morphological features, which will provide a novel venue in studies of microbial pathogenesis. This scheme opens a new route to not only detect bacterial infection but also to other biological tasks.

\section{REFERENCES}

[1] H. C. King et al., "Environmental reservoirs of pathogenic mycobacteria across the Ethiopian biogeographical landscape," PLoS One, vol. 12, no. 3, p. e0173811, 2017.

[2] World Health Organization, "WHO | Global Tuberculosis Report 2017," 2017.

[3] M. B. Conde et al., "Comparison of sputum induction with fiberoptic bronchoscopy in the diagnosis of tuberculosis: Experience at an acquired immune deficiency syndrome reference center in Rio de Janeiro, Brazil," Am. J. Respir. Crit. Care Med., vol. 162, no. 6, pp. 2238-2240, 2000.

[4] K. R. Steingart et al., "Fluorescence versus conventional sputum smear microscopy for tuberculosis: a systematic review," Lancet Infect. Dis., vol. 6, no. 9, pp. 570-581, 2006.

[5] L. E. Cuevas et al., "A multi-country non-inferiority cluster randomized trial of frontloaded smear microscopy for the diagnosis of pulmonary tuberculosis," PLoS Med., vol. 8, no. 7, p. e1000443, 2011.

[6] E. H. Robin, F. S. Maybelle, and B. B. John, "The Tuberculin Skin Test," Clin. Infect. Dis., vol. 17, no. No. 6, pp. 968-975, 1993.

[7] E. Chiappini et al., "Interferon- $\gamma$ release assays for the diagnosis of mycobacterium tuberculosis infection in children: A systematic review and meta-analysis," International Journal of Immunopathology and Pharmacology. pp. 557-564, 2012.

[8] C. C. Boehme et al., "Feasibility, diagnostic accuracy, and effectiveness of decentralised use of the Xpert MTB/RIF test for diagnosis of tuberculosis and multidrug resistance: A multicentre implementation study," Lancet, vol. 377.9776, pp. 1495-1505, 2011.

[9] V. Kumar et al., "Radiomics: The process and the challenges," Magn. Reson. Imaging, vol. 30, no. 9, pp. 1234-1248, 2012.

[10] H.-C. Shin et al., "Deep Convolutional Neural Networks for ComputerAided Detection: CNN Architectures, Dataset Characteristics and Transfer Learning," IEEE Trans. Med. Imaging, vol. 35, no. 5, p. 1285, Feb. 2016.

[11] W. Sun, B. Zheng, and W. Qian, "Computer aided lung cancer diagnosis with deep learning algorithms," SPIE Med. Imaging, vol. 9785, p. 97850Z-97850Z-8, 2016.

[12] W. Du et al., "Breast MR imaging with high spectral and spatial resolutions: preliminary experience," Radiology, vol. 224, pp. 577-585, 2002.

[13] W. Sun, T. B. Tseng, J. Zhang, and W. Qian, "Enhancing deep convolutional neural network scheme for breast cancer diagnosis with unlabeled data," Comput. Med. Imaging Graph., vol. 57, pp. 4-9, 2017.

[14] S. Xuejun, Q. Wei, and S. Dansheng, "Ipsilateral-mammogram computeraided detection of breast cancer," Comput. Med. Imaging Graph., vol. 28, no. 3, pp. 151-158, 2004.

[15] J. Kleesiek et al., "Deep MRI brain extraction: A 3D convolutional neural network for skull stripping," Neuroimage, vol. 129, pp. 460-469, 2016. 
[16] E. Hosseini-Asl, R. Keynton, and A. El-Baz, "Alzheimer's disease diagnostics by adaptation of 3D convolutional network," Proc. - Int. Conf. Image Process. ICIP, vol. 2016-Augus, no. 502, pp. 126-130, 2016.

[17] D. Ciresan, A. Giusti, L. Gambardella, and J. Schmidhuber, "Deep Neural Networks Segment Neuronal Membranes in Electron Microscopy Images," 2012 Conf. Neural Inf. Process. Syst., pp. 2843-2851, 2012.

[18] S. D. Krauß, R. Roy, H. K. Yosef, K. Gerwert, and A. Mosig, "Hierarchical deep convolutional neural networks combine spectral and spatial information for highly accurate Raman-microscopy-based cytopathology," J. Biophotonics, no. May, p. e201800022, 2018.

[19] H. Greenspan, B. van Ginneken, and R. M. Summers, "Guest Editorial Deep Learning in Medical Imaging: Overview and Future Promise of an Exciting New Technique," IEEE Trans. Med. Imaging, vol. 35, no. 5, pp. 1153-1159, 2016.

[20] A. Krizhevsky, I. Sutskever, and G. E. Hinton, "ImageNet Classification with Deep Convolutional Neural Networks," in Proc. \{NIPS\}, 2012.

[21] K. Simonyan and A. Zisserman, "Very Deep Convolutional Networks for Large-Scale Image Recognition," arXiv:1409.1556, 2014.

[22] W. Cong, X. Intes, and G. Wang, "Reconstruction of optical parameters for molecular tomographic imaging," Jul. 2017.

[23] S. Christodoulidis, M. Anthimopoulos, L. Ebner, A. Christe, and S. Mougiakakou, "Multisource Transfer Learning with Convolutional Neural Networks for Lung Pattern Analysis," IEEE J. Biomed. Heal. Informatics, vol. 21, no. 1, pp. 76-84, 2017.

[24] A. K. Barczak et al., "Systematic, multiparametric analysis of Mycobacterium tuberculosis intracellular infection offers insight into coordinated virulence," PLoS Pathog., vol. 13, no. 5, p. e1006363, 2017.

[25] P. Tao, "Detect circles with various radii in grayscale image via Hough transform," University of Maryland, Maryland, 2006.

[26] Q. Zhang et al., "EsxA membrane-permeabilizing activity plays a key role in mycobacterial cytosolic translocation and virulence: Effects of singleresidue mutations at glutamine 5," Sci. Rep., vol. 6, p. 32618, 2016.

[27] Y. Jia, " $\{$ Caffe $\}$ : An Open Source Convolutional Architecture for Fast Feature Embedding." 2013.

[28] P. V. C. Hough, "Method and means for recognizing complex patterns," 1962.

[29] H. Yuen, J. Princen, J. Illingworth, and J. Kittler, "Comparative study of Hough Transform methods for circle finding," Image Vis. Comput., vol. 8, no. 1, pp. 71-77, 1990.

[30] Y. Wang and G. Cheng, "Application of gradient-based Hough transform to the detection of corrosion pits in optical images," Appl. Surf. Sci., vol. 366, pp. 9-18, 2016.

[31] S. Wu, S. Zhong, and Y. Liu, "Deep residual learning for image steganalysis," Multimed. Tools Appl., vol. 77, no. 9, pp. 10437-10453, 2018.

[32] K. He, X. Zhang, S. Ren, and J. Sun, "Deep Residual Learning for Image Recognition," in 2016 IEEE Conference on Computer Vision and Pattern Recognition (CVPR), 2016, pp. 770-778.

[33] N. Tajbakhsh et al., "Convolutional Neural Networks for Medical Image Analysis: Full Training or Fine Tuning?," IEEE Trans. Med. Imaging, vol. 35, no. 5, pp. 1299-1312, 2016.

[34] M. D. Zeiler, G. W. Taylor, and R. Fergus, "Adaptive Deconvolutional Networks for Mid and High Level Feature learning," in 2011 IEEE International Conference on. IEEE, 2011, pp. 2018-2025.

[35] S. Jost Tobias, D. Alexey, B. Thomas, and R. Martin, "Striving for simplicity: The all convolutional net," arXiv:1412.6806, 2014.

[36] V. Dumoulin and F. Visin, "A guide to convolution arithmetic for deep learning," arXiv:1603.07285, 2016.

[37] J. Yosinski, J. Clune, A. Nguyen, T. Fuchs, and H. Lipson, "Understanding Neural Networks Through Deep Visualization," arXiv:1506.06579, Jun. 2015.

[38] K. A. McDonough, Y. Kress, and B. R. Bloom, "Pathogenesis of tuberculosis: Interaction of Mycobacterium tuberculosis with macrophages," Infect. Immun., vol. 61, no. 7, pp. 2763-2773, 1993.

[39] D. J. Kusner and J. A. Barton, "ATP Stimulates Human Macrophages to Kill Intracellular Virulent Mycobacterium tuberculosis Via CalciumDependent Phagosome-Lysosome Fusion," J. Immunol., vol. 167, no. 6, pp. 3308-3315, 2001.

[40] Z. A. Malik, S. S. Iyer, and D. J. Kusner, "Mycobacterium tuberculosis Phagosomes Exhibit Altered Calmodulin-Dependent Signal Transduction Contribution to Inhibition of Phagosome-Lysosome Fusion and Intracellular Survival in Human Macrophages," J. Immunol., vol. 166, no. 5, pp. 3392-3401, 2001.

[41] M. Podinovskaia, W. Lee, S. Caldwell, and D. G. Russell, "Infection of macrophages with Mycobacterium tuberculosis induces global modifications to phagosomal function," Cell. Microbiol., vol. 15, no. 6, pp. 843-859, 2013.

[42] M. V. S. Rajaram et al., "M. tuberculosis-Initiated Human Mannose Receptor Signaling Regulates Macrophage Recognition and Vesicle Trafficking by FcR $\gamma$-Chain, Grb2, and SHP-1," Cell Rep., vol. 21, no. 1, pp. 126-140, 2017.

[43] L. M. Stamm et al., "Mycobacterium marinum Escapes from Phagosomes and Is Propelled by Actin-based Motility," J. Exp. Med., vol. 98, no. 9, pp. 1361-1368, 2003.

[44] L. Y. Gao, S. Guo, B. McLaughlin, H. Morisaki, J. N. Engel, and E. J. Brown, "A mycobacterial virulence gene cluster extending RD1 is required for cytolysis, bacterial spreading and ESAT-6 secretion," Mol. Microbiol., vol. 53, no. 6, pp. 1677-1693, 2004.

[45] R. Simeone et al., "Phagosomal rupture by Mycobacterium tuberculosis results in toxicity and host cell death," PLoS Pathog., vol. 8, p. e1002507, 2012.

[46] Y. Ito, W. Ohyama, T. Wakabayashi, and F. Kimura, "Detection of Eyes by Circular Hough Transform and Histogram of Gradient," Int. Conf. Pattern Recognit., 2012.

[47] O. Barinova, V. Lempitsky, and P. Kholi, "On detection of multiple object instances using hough transforms," IEEE Trans. Pattern Anal. Mach. Intell., vol. 34, no. 9, pp. 1773-1784, 2012.

[48] O. Ronneberger, P. Fischer, and T. Brox, "U-Net: Convolutional Networks for Biomedical Image Segmentation," in International Conference on Medical image computing and computer-assisted intervention, 2015, pp. 234-241.

[49] D. Song et al., "Quantificational and Statistical Analysis of the Differences in Centrosomal Features of Untreated Lung Cancer Cells and Normal Cells," Anal Quant Cytol Histol, vol. 32, pp. 280-290, 2010.

[50] P. Wang, X. Hu, Y. Li, Q. Liu, and X. Zhu, "Automatic cell nuclei segmentation and classification of breast cancer histopathology images," Signal Processing, vol. 122, pp. 1-13, 2016.

[51] L. Clarke, Q. Wei, and K. Maria, "Nuclear imaging enhancer," No. $5,576,548,1996$.

[52] S. Wenqing, "Deep Learning Method vs. Hand-Crafted Features for Lung Cancer Diagnosis and Breast Cancer Risk Analysis," The University of Texas at El Paso, 2017.

[53] M. Xu, D. P. Papageorgiou, S. Z. Abidi, M. Dao, H. Zhao, and G. E. Karniadakis, "A deep convolutional neural network for classification of red blood cells in sickle cell anemia," PLoS Comput. Biol., vol. 13, no. 10, p. e1005746, 2017.

[54] C. L. Chen, A. Mahjoubfar, L. Tai, I. K. Blaby, and A. Huang, "Deep Learning in Label-free Cell Classification," Sci. Rep., vol. 6, p. 21471, 2016.

[55] C. Szegedy et al., "Going Deeper with Convolutions," arXiv:1409.4842, 2014. 